\title{
Miranda
}

Revue pluridisciplinaire du monde anglophone /

Multidisciplinary peer-reviewed journal on the English-

speaking world

$12 \mid 2016$

Mapping gender. Old images; new figures

\section{Double jeu, jeu de doubles : entre Shakespeare et Wilde}

Entretien avec Pieryk Vanneuville

Nathalie Vincent-Arnaud

\section{OpenEdition}

Édition électronique

URL : http://journals.openedition.org/miranda/8318

DOI : 10.4000/miranda.8318

ISSN : 2108-6559

Éditeur

Université Toulouse - Jean Jaurès

Référence électronique

Nathalie Vincent-Arnaud, «Double jeu, jeu de doubles : entre Shakespeare et Wilde », Miranda [En ligne], 12 | 2016, mis en ligne le 29 février 2016, consulté le 16 février 2021. URL : http:// journals.openedition.org/miranda/8318; DOI : https://doi.org/10.4000/miranda.8318

Ce document a été généré automatiquement le 16 février 2021.

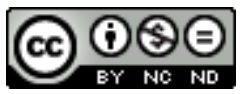

Miranda is licensed under a Creative Commons Attribution-NonCommercial-NoDerivatives 4.0 International License. 


\section{Double jeu, jeu de doubles : entre Shakespeare et Wilde}

Entretien avec Pieryk Vanneuville

Nathalie Vincent-Arnaud

\section{Introduction}

1 En 2015, en l'espace de trois mois, deux pièces majeures du répertoire anglo-saxon, Il importe d'être constant d'Oscar Wilde, suivi de Hamlet, ont été mises à l'honneur dans la programmation du théâtre. Le déploiement d'une scénographie inventive et efficace, la mise en valeur des deux textes au moyen d'un jeu sensible et nourri d'une réflexion poussée sur les résonances contemporaines des deux pièces ne sont pas les moindres ingrédients de la réussite esthétique et expressive de l'ensemble.

\section{Pieryk Vanneuville: biographie}

2 Pieryk Vanneuville est comédien et metteur en scène au Théâtre du Jour à Agen, qui comprend le Théâtre École d'Aquitaine dont il est directeur adjoint chargé des études et la Compagnie Pierre Debauche.

\section{Il importe d'être constant; Hamlet: fiches spectacles}

\section{Il importe d'être constant}

Création : mai 2015, Théâtre du Jour (http://www.theatredujour.com/)

Production : Théâtre Ecole d'Aquitaine (http://www.theatredujour.com/theatre-ecoledaquitaine-3/), Compagnie Pierre Debauche

Texte : Oscar Wilde ; Traduction : Robert Angebaud

Mise en scène : Pieryk Vanneuville

Assistant à la mise en scène : Eva Guionnet 
Avec Simon Alteirac, Iysha Barton, Christophe Caulet, Françoise Danelle, Vincent Danelle, Vincent Davoux, Agnes François, Valentine Regnaut, Timour Van Der Bilj, Pieryk Vanneuville

Hamlet

Création : juillet 2015, Théâtre du Jour (http://www.theatredujour.com/)

Production: Théâtre Ecole d'Aquitaine (http://www.theatredujour.com/theatre-ecoledaquitaine-3/), Compagnie Pierre Debauche

Texte : William Shakespeare ; Traduction : Robert Angebaud

Metteur en scène : Pieryk Vanneuville

Assistant à la mise en scène : Mathilde Garrat

Dramaturgie : Robert Angebaud

Avec Myke Alias, Robert Angebaud, Joy Bernard, Nolwenn Bertrand, Clémence Biensan, Christophe Caulet, Adeline Chaigne, Noémie Corlardeau, Olivier Dumas, Charlie Duval, Elise Ghienne, Alexandre Giet, Louise Grenier, Giulia Grosman, Dorian Lopes, Marine Manfredi, Lucas Manrique, Sache Pointevin, Valentine Regnaut, Timour Van der Bijl, Pieryk Vanneuville

\section{Entretien}

Nathalie Vincent-Arnaud: Le Théâtre du Jour a mis au programme, en un laps de temps très réduit, Hamlet (en ouverture du festival de théâtre d'Agen en juillet 2015) et, un peu plus tôt, Il importe d'être constant. Comme tu me l'as dit, il s'agit d'un hasard de programmation. $Y$ a-t-il des raisons particulières au choix d'une pièce de Wilde?

Pieryk Vanneuville: On souhaitait monter une comédie pour le printemps, époque à laquelle le public sort de la torpeur de l'hiver et recherche avant tout le rire. Robert Angebaud connaissait et aimait cette pièce et avait envie de la monter depuis longtemps. Il m'a donc demandé de jouer Jack, ou plutôt Constant, enfin... les deux ! Il est vrai aussi que j'ai personnellement un fort tropisme pour la culture anglaise et pour le XIXè siècle anglais. J'ai déjà adapté Oliver Twist, Peter Pan (en comédie musicale), L'T̂le au trésor, et j'aimerais énormément adapter Alice au pays des Merveilles. Et en dehors du XIXè, Shakespeare reste un de mes auteurs préférés et je n'en ai certainement pas fini avec lui.

NVA : On l'espère bien. Existe-t-il selon toi des traits communs (stylistiques, thématiques) au théâtre anglo-saxon dans son ensemble qui conditionnent son approche par un metteur en scène et un comédien?

PV : Une chose ressort vraiment : l'humour. Il y a vraiment un style d'humour propre au théâtre anglais, très cruel, beaucoup plus cinglant que dans une comédie française (toutes époques confondues). Si l'on considère Shakespeare et Wilde, on peut retrouver chez Shakespeare une utilisation du double constamment présente; chez Wilde aussi sans doute, mais sans l'aspect baroque que seul shakespeare développe (même si l'on trouve cela chez Marlowe aussi, auquel je compte bien m'attaquer un jour, même s'il y a des différences très importantes entre les deux auteurs). Ce qu'on trouve aussi dans le théâtre anglais, et qui n'existe pas autant dans le théâtre français, c'est le côté policier, qui fait songer aux classiques tels qu'Agatha Christie ou Conan Doyle. Il existe une ambiance anglaise particulière, de menace, de couteau planté dans le dos sous couvert de bienséance. C'est perceptible dans le langage. On joue les pièces en français mais quand je jouais Roméo et Juliette je m'étais attelé à la traduction. Et il $\mathrm{y}$ a tout un jeu de langage, de rythmique très compliqué à faire 
passer en français. Il y a des dramaturges français qui jouent avec les mots bien sûr. Mais dans Roméo par exemple Shakespeare ré-invente le langage pour aboutir à des jeux de mots. Chez Wilde, cela passe par les aphorismes.

NVA: Certaines personnes reprochent parfois à l'écriture de Wilde de présenter un peu toujours la même mécanique, la même recette, les cascades d'aphorismes pouvant engendrer pour eux une forme de lassitude. Quel est ton avis sur cette question?

$P V$ : On pourrait dire la même chose de Guitry, de Feydeau... Il est certain qu'il existe une mécanique de dramaturgie mais il n'empêche que les sujets sont différents même si les univers se ressemblent d'une pièce à l'autre.

NVA: Quel a été l'accueil de ces deux pièces successives (Hamlet et // importe d'être constant) par le public? Qu'est-ce qui, à ton avis, est susceptible de toucher particulièrement les contemporains dans chacune de ces pièces?

PV : L'accueil des deux spectacles a été bon. Dans Il importe d'être constant, le rapport du couple est encore d'aujourd'hui. Qui a le pouvoir, l'homme ou la femme ? C'est la question centrale. Chez Wilde, les femmes mènent vraiment le jeu (voir Gwendoline et Lady Bracknell, et la manière dont elles évoquent les hommes). Ce rapport-là à la société fait toujours écho de nos jours. Quand on monte des pièces, on les monte avec un public d'aujourd'hui, et même lorsqu'on reprend une pièce vieille de plusieurs siècles on la joue pour les gens qui sont en face de nous. En montant Hamlet, je ne peux pas faire comme si rien ne s'était passé depuis. Je vais bien sûr jouer la pièce telle qu'elle a été écrite et ce qu'elle raconte, tout en sachant que je joue pour des gens d'aujourd'hui. Quand je parle de ce problème latent de la guerre et du monde féodal qui est en train de s'écrouler (Fortinbras étant, lui, signe d'un nouveau monde, d'une autre façon de penser la politique), je suis face à des choses qui se passent à l'heure actuelle, une manière de quitter un monde pour aller dans un autre. Cela ne veut pas dire que je vais raconter les massacres survenus depuis, les guerres mondiales, etc. Mais nous acteurs, nous portons le monde d'aujourd'hui dès que nous montons sur un plateau. Pendant la dernière saison, j'ai aussi joué Perdican dans On ne badine pas avec l'amour, juste au moment où sont survenus les attentats de Charlie Hebdo. D'un seul coup, la volonté de Camille d'entrer au couvent, sa vision bloquée dans une religion ont été chargées de résonances contemporaines, qui ont bien sûr transparu sur scène.

NVA: Dans Hamlet, j'ai été frappée par deux éléments de la scénographie: les chorégraphies (ou plutôt les déplacements chorégraphiés) et l'emploi du chant, des chœurs de femmes, des percussions à plusieurs reprises. Peux-tu nous éclairer sur l'emploi de tout cela? Que représentent au juste ces éléments?

$\mathrm{PV}$ : Quand je travaille sur une mise en scène, je travaille avec des corps. Il y a bien sûr un texte, mais le texte ne peut être dit s'il n'est pas investi. C'est une chose qui revient régulièrement quand on me parle de mes spectacles, de cette espèce de chorégraphie constante sur le plateau. Je travaille au préalable sur un espace, celui dans lequel je vais emmener mes acteurs, et je marche par croquis successifs qui vont amener à la répétition. En revanche, je ne vais pas inventer à l'avance ce qui va se passer, je ne peux construire qu'avec les acteurs et ce qu'ils vont me proposer. J'ai un espace, un récit, une fable, des acteurs, et je fais s'entrechoquer ces éléments pour arriver à une construction. J'ai toujours besoin de musique, parfois enregistrée, mais si je peux faire en sorte que la musique soit vivante et produite de manière directe, cela représente une force plus grande à mes yeux et qui me semble inhérente à la nature du théâtre. Parmi les retours qu'on m'a faits de Hamlet, alors que je n'avais pas 
théorisé la chose, il y a eu la remarque : « Cette manière de ramener le chœur antique dans Shakespeare, c'est grandiose ».

NVA : Oui, c'est aussi mon avis.

5 PV : Oui, mais ce n'était pas délibéré et cela s'est fait dans un ensemble de choses. Ces actrices étant présentes avec leurs qualités musicales et chorégraphiques, je me suis servi de cela. J'ai vu qu'elles pouvaient m'apporter l'espace tragique, et c'est là en effet qu'on retrouve le chœur antique. Elles ne sortent quasiment jamais de la scène, leur présence constante évoque le destin. Ce sont elles qui maîtrisent les changements d'espace : quand tout se casse, elles exècutent des gestes, des danses qui amènent vers la fin, vers la mort. Il y a beaucoup de morts chez Shakespeare, et j'en ai encore ajouté [rires]. Et ce chœur donne du relief, en particulier le côté percussif, métallique, qui non seulement est raccordé à ma propre esthétique de scénographie mais en plus apporte vraiment de la puissance. Qui plus est, c'est ce qu'écrit Shakespeare, une des répliques de Fortimbras étant "Faites taire les tambours». On prend donc un élément à un moment donné et on le développe, on l'amplifie. Je suis très sensible au métal, qui dans Shakespeare m'amène l'idée du piège, de prison. Il me permet de construire un espace où la lumière va jouer un rôle important (je travaille beaucoup avec mon éclairagiste), le métal produisant des effets de lumière particuliers, des effets de résonance, des contrastes ombre et lumière. Ce sont tous ces éléments, toutes ces images que l'on va parfois chercher très loin, au fil de ses lectures, et qui reviennent quand on travaille une mise en scène, qui produisent des effets dont on n'était pas conscient. Ce qui est très beau avec Hamlet, qui reste pour moi la pièce centrale de tout le théâtre occidental, c'est que l'on n'a pas fini de soulever les mystères. C'est une boîte de Pandore : on va ouvrir de nombreux tiroirs, sans forcément répondre à toutes les questions, notre rôle en tant qu'acteurs étant de soulever ces questions. Nous reprenons Shakespeare à la prochaine saison, et nous allons certainement encore trouver des choses. C'est la pièce qui m'a fait décider d'être acteur; je l'ai vue 7 fois de suite, c'est ce qui m'a vraiment amené au théâtre. Si l'on prend Musset dans le théâtre français, il est évident qu'il a lu Shakespeare ; c'est le seul dramaturge français qui ait pratiqué ce mélange de genres.

NVA : Je trouve pour ma part que le personnage de Hamlet renferme à lui seul toute la fabrique du théâtre. Hamlet ne cesse de jouer et de faire jouer les autres.

$7 \quad P V$ : Il y a beaucoup de questions à se poser sur le personnage de Hamlet. Combien de temps a-t-il réellement connu son père ? Il a passé la plupart de son temps à étudier en Allemagne. Par ailleurs il ne parvient jamais à prendre une réelle décision. Ophélie, quant à elle, est la proie de tout le monde, d'Hamlet et de son comportement odieux, de son père, de la mère d'Hamlet qui l'enjoint de jouer le rôle qu'on attend d'elle. D'ailleurs, si l'on examine le champ lexical de la pièce, on s'aperçoit qu'il y a énormément de termes qui se rapportent au théâtre, à la scène qu'on doit jouer, au rôle qu'on doit tenir. Tous les personnages utilisent ce langage du théatre, pas forcément au moment de la représentation des comédiens. Lorsque Hamlet prévient Marcellus et Horatio, juste après l'apparition du spectre, qu'il va prendre l'apparence de la folie, il s'apprête à endosser un nouveau costume. Quand on travaille sur la dramaturgie de la pièce, on voit qu'il y a deux champs lexicaux qui dominent : celui du théâtre, et aussi celui de la pourriture, comme si les personnages semblaient constamment s'embourber. «Il y a quelque chose de pourri au royaume du Danemark »... 
NVA: Dans ton interprétation du héros de Shakespeare, tu optes pour une diction très spécifique pour "des mots, des mots, des mots» en détachant chacune des trois séquences tandis que tu te déplaces sur scène de manière facétieuse. Pourquoi ce choix?

$P V$ : C'est vrai, mais il y a aussi «Bien, bien, bien » avec Ophélie et avec Polonius. Hamlet utilise fréquemment le même mot répété trois fois, c'est vraiment un trait d'écriture typique dans la pièce. Pour " des mots... des mots... des mots ", j'opte en effet pour des variations de ton comme si Polonius n'avait pas compris. Le phrasé un peu étrange vient aussi du fait qu'on a essayé de respecter la versification shakespearienne et ses mises en relief. C'est quelque chose de très important quand on travaille la pièce.

NVA: Justement, il existe plusieurs traductions françaises, anciennes et récentes, de chacune des deux pièces. Sur lesquelles t'es-tu appuyé et pour quelles raisons ? Qu'est-ce qui, au Théâtre du Jour, influence votre choix d'une traduction ou d'une autre de manière générale?

PV : Que ce soit Wilde ou Shakespeare, la traduction a été faite par Robert Angebaud. On n'a pas eu recours à une traduction faite par autrui car il y a un intérêt à cela : le fait de faire sa traduction permet de réfléchir à la manière dont la pièce a été écrite en anglais, à la rythmique et à la versification de Shakespeare. De même, dans certaines traductions, il y a des mots qui ne sont pas forcément usités de nos jours. Ainsi, le langage de Roméo et Juliette, le langage des jeunes est très vert ; l'objectif de la traduction n'est pas de trouver un langage "jeune » mais il ne faut pas perdre de vue que lorsque Shakespeare écrit il utilise un langage grossier qui correspond à celui de l'époque (ce que le public oublie la plupart du temps lorsqu'il va voir la pièce). Si, aujourdhui, on dit «faquin » au lieu de «sale con», les gens vont entendre «faquin » comme un mot poétique car le sens véritable du mot a été oublié. Sur Roméo et Juliette, sans pour autant appauvrir le langage, je suis allé rechercher le sens en essayant de retrouver la force du langage. Si le langage est violent, c'est cette violence qu'il faut retrouver au lieu de rechercher à tout prix le littéraire. Shakespeare est incroyable d'audace et de choses mises à mal. La traduction oblige à voir tout cela. Quand on prend des auteurs plus récents, il est vrai qu'on est obligé d'utiliser une traduction existante à cause des droits d'auteur, ce qui oblige parfois à faire des choses impensables qui bloquent parfois la création. Sinon, lorsqu'on affaire à un texte plus ancien, comme Shakespeare, ce travail de traduction oblige à comprendre d'abord l'écriture et d'entendre la musicalité du texte, sa respiration. On revient à la base même de l'acteur qui est le souffle, et le souffle ne se produit que par le corps.

NVA : Revenons un peu à Wilde. I/ importe d'être constant / // importe d'être sérieux : ces deux titres ont été donnés en français à la pièce de Wilde par les différents traducteurs. Le jeu de mot sur le prénom est évidemment une contrainte. Mais sinon, quelle est la pertinence des deux à ton avis?

PV : En fait il y a même eu cinq traductions de l'adjectif, dont «honnête »! Effectivement, ce mélange qu'offre le mot en anglais n'a pas d'équivalent en français. "Constant» est ce qui fonctionnait le mieux aux yeux de Robert Angebaud, indépendamment même du jeu de mot avec le prénom, et je suis d'accord. Cela corrobore aussi l'une des répliques finales de Gwendoline qui utilise la notion de constance. 
NVA : Quels sont les trois adjectifs qui, selon toi, décriraient le mieux le héros de Hamlet? Même question pour le héros de /l importe d'être constant.

PV : Question difficile. Pour Jack, ce serait dandy (si cela peut être un adjectif!), hésitant, et double. Pour Hamlet, ce serait indécis, impulsif (il est irréfléchi même s’il raisonne sans cesse), fidèle...même si cette fidélité ne s'applique pas à tout. Chacun d'entre eux est ambivalent, ne se laisse pas facilement appréhender. C'est une réplique de l'être humain en fait.

NVA: Dans la mise en scène de Wilde que propose Robert Angebaud, les paravents qui ornent les appartements d'Algernon montrent, si j'ai bien vu (de loin...), des dessins d'Aubrey Beardsley. Peux-tu nous en dire plus sur ces dessins?

$P V$ : Je pense qu'il s'agit de dessins faits à l'origine pour Salomé, et très importants dans l'univers de Wilde. On a voulu concevoir trois lieux distincts, et pour celui-ci je me suis imaginé la chambre d'étudiant de Wilde, assez étrange. Ces dessins, par leur caractère scandaleux et un peu sanglant, étaient appropriés.

NVA: Quelques mots encore, peut-être, sur les personnages de Wilde?

PV : Le rôle central chez Wilde est Lady Bracknell. C'est un rôle qui pourrait tout à fait être joué par un homme, et c'est d'ailleurs un point de ressemblance avec Shakespeare : par exemple, la nourrice dans Roméo et Juliette pourrait elle aussi être interprétée par un homme. Il s'agit de personnages très forts qui semblent parfois être davantage conçus pour un acteur que pour une actrice. Lady Bracknell en homme serait sans doute grandiose, comme d'ailleurs d'autres personnages, chez Molière par exemple, où certains rôles sont empreints de force et de masculinité. Un homme pourrait être amené à les interpréter en recherchant en lui une part féminine. Mais bien sûr il s'agit d'un choix d'esthétique et de mise en scène.

\section{RÉSUMÉS}

Entretien avec Pieryk Vanneuville. L'entretien fut conduit par Nathalie Vincent-Arnaud le 10 septembre 2015 au Café de la Bourse (Agen, France)

Interview with Pieryk Vanneuville. The interview by Nathalie Vincent took place on September, $10^{\text {th }}$ at the Café de la Bourse (Agen, France)

\section{INDEX}

Thèmes: Theater

Keywords : body, playwriting, language, scenography, theatre

Mots-clés : corps, dramaturgie, langage, scénographie, théâtre 


\section{AUTEURS}

\section{NATHALIE VINCENT-ARNAUD}

Université Toulouse-Jean Jaurès

Professeur

nathalie.vincentarnaud@sfr.fr 